\title{
Effect of Date of Sowing and Foliar Application of Nutrients on Seed Quality of Soybean
}

\author{
G.M. Sumalatha* and D.S. Uppar \\ Department of Seed Science and Technology, College of Agriculture, Dharwad, University of \\ Agricultural Sciences, Dharwad, India \\ *Corresponding author
}

\begin{tabular}{|l|}
\hline Ke y w o r d s \\
$\begin{array}{l}\text { Sowing and foliar } \\
\text { application, } \\
\text { Nutrients, Seed } \\
\text { Quality, Soybean }\end{array}$ \\
\hline Article Info \\
\hline $\begin{array}{l}\text { Accepted: } \\
\text { 06 July 2018 } \\
\text { Available Online: } \\
\text { 10 August } 2018\end{array}$ \\
\hline
\end{tabular}

\section{A B S T R A C T}

An experiment was conducted to study the influence of sowing dates and foliar application of nutrients on seed quality parameters of soybean variety DSb-21 during kharif, 2016 and 2017. Sowings were taken up at fortnight interval they are, first fortnight of June, second fortnight of June and First fortnight of July. The seeds from field experiment were subjected to lab analysis, the results of the experiment revealed that the above parameters significantly affected by sowing dates and foliar application of nutrients. Among the dates of sowing, first fortnight of June $\left(D_{1}\right)$ recorded highest values for test weight (13.69 $\mathrm{g}$ ), germination $(95.35 \%)$, root length $(17.39 \mathrm{~cm})$, shoot length $(17.06 \mathrm{~cm})$, seedling dry weight $(124.0 \mathrm{mg})$, seedling vigour index-I (3282), germination rate index (64.05), total dehydrogenase activity ( 0.614 OD value), oil content $(18.13 \%)$, protein content $(37.59 \%)$ and lower seed infection (14.24\%) compared to other dates of sowing. Foliar spray of $\mathrm{KNO}_{3} @ 0.5 \%+\mathrm{KH}_{2} \mathrm{PO}_{4} @ 0.5 \%+$ Boron $0.50 \%$ recorded highest test weight (13.54 g), germination $(96.28 \%)$, root length $(17.35 \mathrm{~cm})$, shoot length $(17.48 \mathrm{~cm})$, seedling dry weight $(123.7 \mathrm{mg})$, seedling vigour index-I (3353), germination rate index (64.08), total dehydrogenase activity ( 0.606 OD value), oil content $(18.10 \%)$, protein content $(37.93 \%)$ and lower seed infection (17.03\%) compared to other foliar treatments based on pooled analysis. So the seeds obtained from the crop sown on first fortnight of June sprayed with $\mathrm{KNO}_{3} @ 0.5 \%+\mathrm{KH}_{2} \mathrm{PO}_{4} @ 0.5 \%+$ Boron $0.50 \%$ had the good quality seeds.

\section{Introduction}

Soybean [Glycine max (L.) Merrill] crop is originated in China and distributed to Asia, USA, Brazil, Argentina etc. It is synonymously called as 'Chinese pea' or 'Manchurian bean' or "Golden bean" and it is emerged as a miracle crop of 20th century because it is versatile and fascinating crop. Apart from high yielding potential (30-35 $\mathrm{q} / \mathrm{ha}$ ), soybean is very rich in protein $(40 \%)$ and edible oil (20\%) contains a fairly high amount of unsaturated fatty acids and about 1.5 to 3.1 per cent lecithin which is essential for building up of nerve tissue. Soybean is the single largest oilseed produced in the world. It alone contributes about 58 per cent of the global oil seed production. It ranks first in oil seed production followed by rapeseed (13\%), groundnut (8\%) and sunflower (7\%). Globally, soybean occupies an area of $126.6 \mathrm{~m}$ ha producing $346.3 \mathrm{mt}$ with the productivity of 
$2735 \mathrm{~kg}$ per ha. In India soybean occupies an area of $10.60 \mathrm{~m}$ ha producing $12.22 \mathrm{~m} . \mathrm{t}$ with productivity of $1153 \mathrm{~kg}$ per ha and Karnataka with an area of $0.27 \mathrm{~m}$ ha producing $0.17 \mathrm{~m} . \mathrm{t}$ with productivity of $639 \mathrm{~kg}$ per ha (Anon., 2017).

Climatic factors like temperature, precipitation or rain, snow fall, wind, wind storms, flooding etc., have crucial role in agricultural production. In agriculture both temperature and precipitation are dominant climatic factors to affect crop yields which vary widely throughout the year and place (Alexandrov and Hoogenboom, 2001). Planting prior to or later than the optimal planting date can greatly reduce soybean yield and quality since photo periodism controls not only the number of days to flowering, but also the amount of time available for vegetative plant growth and development. Soybeans planted prior or late to optimum range often lose yield from poor emergence due to inadequate soil temperature or, when planted after the optimal range, from failure to fully develop (Bastidas et al., 2008).

To increase the productivity of soybean, it is necessary to provide adequate nutrition to the plant for growth and development. Plant nutrition plays an important role for enhancing seed yield and quality in soybean. Foliar application of nutrients was more beneficial than soil application, since application rates are lesser as compared to soil application, same results could be obtained easily and the crop reacts to nutrient application immediately (Zayed et al., 2011).

Recently, new generation fertilizers have been introduced exclusively for foliar feeding and fertilization. These fertilizers are better source for foliar application (Vibhute, 1998). These fertilizers have different ratios of $\mathrm{N}, \mathrm{P}$ and $\mathrm{K}$ which are highly water soluble and so amenable for foliar nutrition (Jayabal et al., 1999). Quality seed production in soybean is holistic approach which involves the activities like standardization of appropriate season, time of planting and other several techniques to enhance the storability. Keeping all these aspects in view, the present investigation was undertaken.

\section{Materials and Methods}

The Field experiment was conducted during kharif season of 2016 and 2017 in MARS, University of agricultural Sciences, Dharwad. The factors of the experiment was comprised of three date of sowing $\left(\mathrm{D}_{1}: 1^{\text {st }}\right.$ fortnight of June, $\mathrm{D}_{2}: 2^{\text {nd }}$ fortnight of June and $\mathrm{D}_{3}: 1^{\text {st }}$ fortnight of July) and eight foliar spray at 45 DAS $\left(\mathrm{T}_{1}\right.$ : Water spray, $\mathrm{T}_{2}$ : Urea spray @ $2 \%$, $\mathrm{T}_{3}$ : Diammonium phosphate (DAP) @ $2 \%$, $\mathrm{T}_{4}$ : Potassium phosphate $\left(\mathrm{KH}_{2} \mathrm{PO}_{4}\right) @ 1 \%$, $\mathrm{T}_{5}$ : Boron@ 0.50\%, $\mathrm{T}_{6}$ : 19:19:19@3\%+ Boron @ $0.50 \%, \mathrm{~T}_{7}: \mathrm{KNO}_{3} @ 1 \%+\mathrm{KH}_{2} \mathrm{PO}_{4}$ @ $0.5 \%$ and $\mathrm{T}_{8}: \mathrm{KNO}_{3} @ 0.5 \%+\mathrm{KH}_{2} \mathrm{PO}_{4} @$ $0.5 \%+$ Boron $0.50 \%$ ) for DSb-21 variety of soybean. The fortnight meteorological observations during crop growth period are presented in Table 1. The seed materials from the field experiment were harvested and subjected for laboratory analysis using Completely Randomized Design with factorial concept in three replications to assess the seed quality parameters in laboratory of National Seed Project, Seed Unit and Department of Seed Science and Technology, University of Agricultural Sciences, Dharwad.

\section{Results and Discussion}

The test weight showed significant difference due to different dates of sowing (Table 2). Higher test weight was recorded in seeds sown on first fortnight of June $\left(D_{1}\right)(13.74,13.64$ and $13.69 \mathrm{~g}$ ) followed by second fortnight of June $\left(\mathrm{D}_{2}\right)$ recorded $13.53,13.40$ and $13.47 \mathrm{~g}$ while, lower test weight was recorded in plots sown on first fortnight of July $\left(\mathrm{D}_{3}\right)$ (13.25, 13.03 and $13.14 \mathrm{~g}$ ) during 2016, 2017 and 
pooled data, respectively. Seed weight significantly declined when sowing was delayed from June to July. The highest test weight recorded in first date of sowing might be due to optimum climate and sufficient time for growth and pod filling, which enabled the plants to produce bold seeds, late sowings decreased the rate of pod filling and shortened effective duration of pod filling compared with earlier sowings. Reduction in the source of assimilates at later sowing dates caused lower seed weight at harvest (Khan, 2001).

These results are in accordance with the findings of several other workers (Yari et al., 2013 and Rahman et al., 2006), who reported that earlier sowings provided the short vegetative period of growth and comparatively longer reproductive and seed filling period, which significantly raised test weight.

Non-significant variations realized for standard germination per cent among different sowing dates (Table 2). Germination ranged from 93 to 97 per cent with 96 per cent as general mean. The highest germination per cent was recorded in seeds obtained from $\left(D_{1}\right)$ first fortnight of June (96.01, 94.48 and 95.25 $\%)$ and lowest was observed in $\left(\mathrm{D}_{3}\right)$ July first fortnight of sowing (95.24, 94.23 and 94.74 $\%$ ) during 2016, 2017 and pooled data, respectively. Higher germination $(95.25 \%)$ in case of June first fortnight of sowing might be due better environment conditions i.e., optimum temperature $\left(23.68{ }^{\circ} \mathrm{C}\right)$ during seed filling, seed development, maturity and harvesting. The lowest germination (94.74\%) in seeds obtained from July first fortnight sowing might be due to the improper growth and development of plants and crop exposed to low mean temperature during maturity and harvesting stage and seeds obtained from these sowings had relatively higher moisture content and fungal infection. These findings are in conformity with Uem and Unioeste (2003) they reported that seeds obtained from the optimum sowing dates had higher per cent of germination than early or delayed planted crop because of the more favourable climatic conditions during seed development maturity.

The root length had significant difference due to different dates of sowing (Table 2). Significantly higher root length (17.40, 17.38 and $17.39 \mathrm{~cm}$ ) was recorded in seeds sown on first fortnight of June $\left(\mathrm{D}_{1}\right)$ followed by second fortnight of June $\left(\mathrm{D}_{2}\right)$ recorded 17.18, 17.09 and $17.14 \mathrm{~cm}$ while, lower root length (16.82, 16.63 and $16.72 \mathrm{~cm}$ ) was recorded in plots sown on first fortnight of July $\left(\mathrm{D}_{3}\right)$ during 2016, 2017 and pooled data, respectively. The results showed maximum shoot length (17.08 $\mathrm{cm}$ ) in case of second date of sowing done at June second fortnight $\left(\mathrm{D}_{2}\right)$ in 2016, $(17.06 \mathrm{~cm})$ in June first fortnight $\left(D_{1}\right)$ during 2017 and pooled data respectively and significantly lower shoot length $(16.49,16.31$ and 16.40 $\mathrm{cm})$ was observed when sowing performed on July first fortnight $\left(\mathrm{D}_{3}\right)$ during 2016, 2017 and pooled data, respectively (Table 2 ).

The significant increase in shoot and root length might be due to higher seed index as is reflected in the test weight of the seed, which might have supplied adequate food reserves to resume embryo growth. These findings are supported by Kumar et al., (2011) who reported that in niger seed quality parameters like germination percentage and seedling length were observed to be lower when the sowing date was delayed.

There was continuous decrease in dry weight of seedlings as sowing date delayed (Table 3 ). Sowing on first fortnight of June $\left(D_{1}\right)$ recorded significantly higher (124.0, 121.9 and $124.0 \mathrm{mg}$ ) dry weight of seedlings followed by second fortnight of June $\left(D_{2}\right)$ recorded 123.4, 120.4 and $123.0 \mathrm{mg}$ and lowest (121.3, 117.2 and $120.3 \mathrm{mg}$ ) was recorded in $\left(\mathrm{D}_{3}\right)$ first fortnight of July during 2016, 2017 and pooled data, respectively. 
Fig.1 Effect of date of sowing and foliar application of nutrients on total dehydrogenase activity of soybean

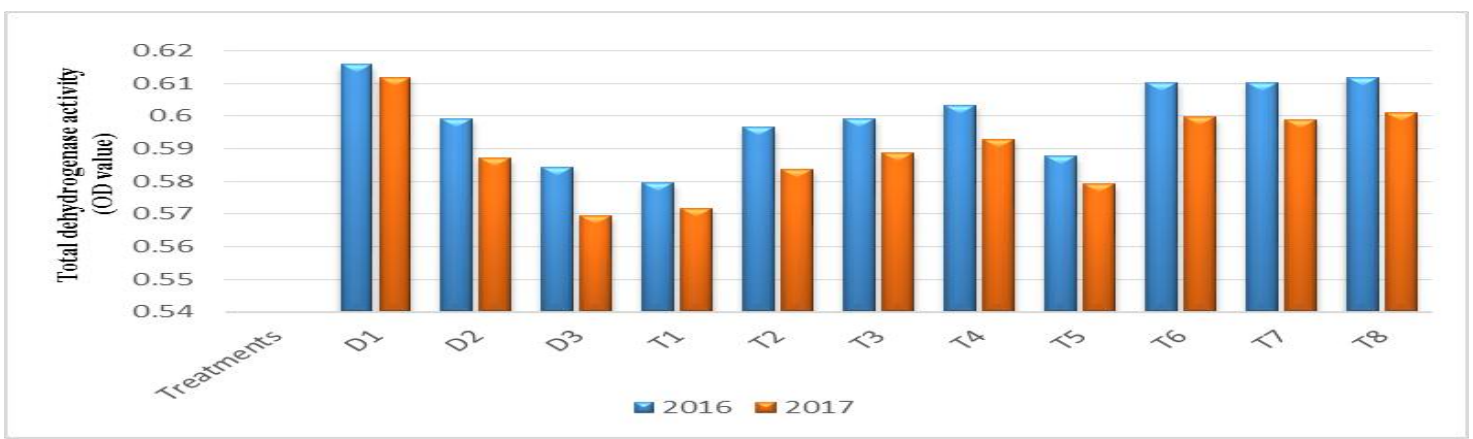

Fig.2 Effect of date of sowing and foliar application of nutrients on oil and protein content of soybean

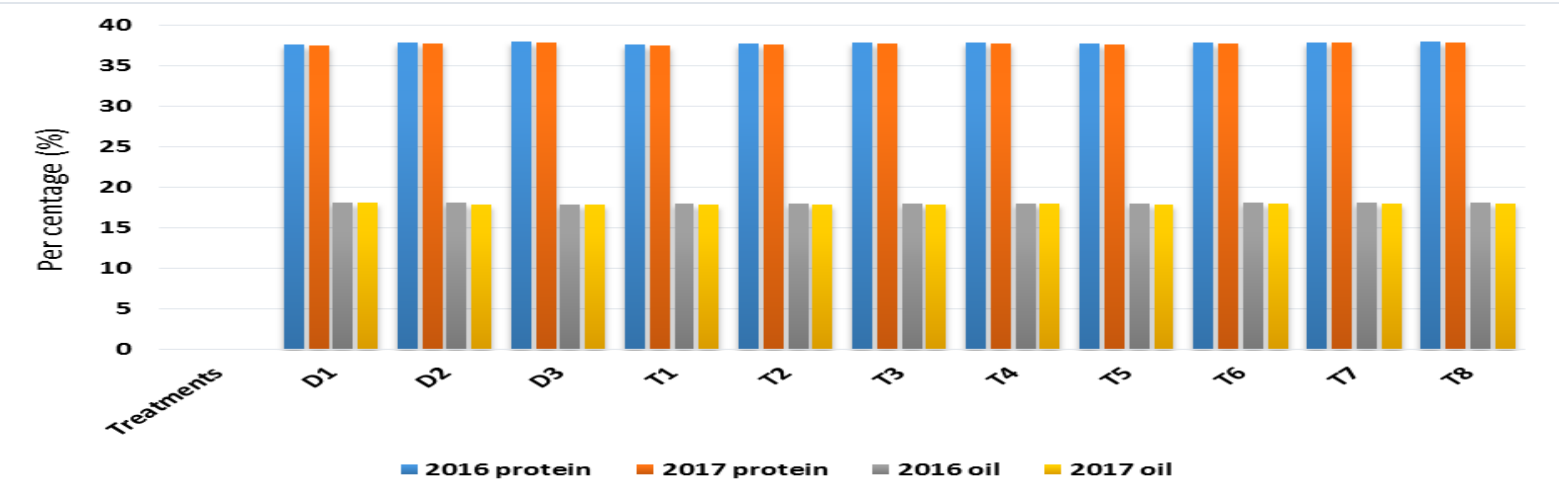

Table.1 Fortnight distribution of minimum and maximum temperature, relative humidity and rainfall during crop growth period over two years

\begin{tabular}{|l|}
\hline Months \\
\hline June 1-15 \\
\hline June 16-30 \\
\hline July 1-15 \\
\hline July 16-31 \\
\hline August 1-15 \\
\hline August 16-31 \\
\hline September 1-15 \\
\hline September 16-30 \\
\hline October 1-15 \\
\hline \hline October 16-31 \\
\hline Total \\
\hline
\end{tabular}

\begin{tabular}{|c|c|c|c|c|c|c|c|c|c|}
\hline \multicolumn{5}{|c|}{2016} & \multicolumn{5}{|c|}{2017} \\
\hline \multicolumn{2}{|c|}{$\begin{array}{c}\text { Mean } \\
\text { temperature }(\mathrm{o} \text { C) }\end{array}$} & \multicolumn{2}{|c|}{$\begin{array}{c}\text { Relative } \\
\text { humidity (\%) }\end{array}$} & \multirow[t]{2}{*}{$\begin{array}{c}\text { Rainfall } \\
\text { (mm) }\end{array}$} & \multicolumn{2}{|c|}{$\begin{array}{c}\text { Mean } \\
\text { temperature (o C) }\end{array}$} & \multicolumn{2}{|c|}{$\begin{array}{c}\text { Relative } \\
\text { humidity }(\%)\end{array}$} & \multirow[t]{2}{*}{ Rainfall (mm) } \\
\hline Max & Min & Max & Min & & Max & Min & Max & Min & \\
\hline 30.1 & 21.6 & 85.7 & 68.2 & 3.1 & 29.7 & 21.5 & 85.9 & 71.2 & 1.1 \\
\hline 28.2 & 21.1 & 86.6 & 75.3 & 1.9 & 29.3 & 21.5 & 85.9 & 72.9 & 1.0 \\
\hline 26.1 & 21.4 & 90.6 & 84.0 & 6.6 & 28.3 & 21.0 & 86.5 & 76.0 & 1.6 \\
\hline 26.5 & 20.6 & 92.1 & 83.1 & 3.2 & 27.0 & 21.1 & 89.6 & 81.3 & 5.9 \\
\hline 25.6 & 20.7 & 92.2 & 83.7 & 4.9 & 28.9 & 20.7 & 87.4 & 73.3 & 0.5 \\
\hline 27.2 & 20.5 & 89.1 & 78.6 & 2.4 & 28.3 & 20.9 & 92.7 & 87.4 & 1.5 \\
\hline 27.4 & 19.8 & 86.6 & 74.9 & 0.8 & 30.0 & 21.2 & 93.1 & 85.5 & 7.5 \\
\hline 26.6 & 20.2 & 89.9 & 83.6 & 4.1 & 27.5 & 20.1 & 93.7 & 91.1 & 5.7 \\
\hline 28.3 & 19.6 & 87.2 & 71.1 & 3.0 & 29.1 & 21.1 & 94.8 & 87.1 & 4.0 \\
\hline 31.1 & 18.0 & 62.5 & 39.2 & 0.0 & 29.9 & 18.8 & 91.6 & 81.4 & 0.8 \\
\hline & & & & 30.0 & & & & & 29.6 \\
\hline
\end{tabular}


Table.2 Effect of date of sowing and foliar application of nutrients on seed weight, seed germination, root length and shoot length of soybean

\begin{tabular}{|c|c|c|c|c|c|c|c|c|c|c|c|c|}
\hline \multirow{2}{*}{$\begin{array}{l}\text { Treatments } \\
\text { Dates of sowing (D) }\end{array}$} & \multicolumn{3}{|c|}{ Seed weight (g) } & \multicolumn{3}{|c|}{ Seed germination $(\%)$} & \multicolumn{3}{|c|}{ Root length (cm) } & \multicolumn{3}{|c|}{ Shoot length (cm) } \\
\hline & 2016 & 2017 & Pooled & 2016 & 2017 & Pooled & 2016 & 2017 & Pooled & 2016 & 2017 & Pooled \\
\hline $\mathbf{D}_{1}$ & 13.74 & 13.64 & 13.69 & 96.01 & 94.48 & 95.25 & 17.40 & 17.38 & 17.39 & 17.06 & 17.06 & 17.06 \\
\hline $\mathbf{D}_{2}$ & 13.53 & 13.40 & 13.47 & 95.62 & 94.301 & 94.96 & 17.18 & 17.09 & 17.14 & 17.08 & 16.93 & 17.00 \\
\hline $\mathbf{D}_{3}$ & 13.25 & 13.03 & 13.14 & 95.24 & 94.23 & 94.74 & 16.82 & 16.63 & 16.72 & 16.49 & 16.31 & 16.40 \\
\hline S. Em. \pm & 0.03 & 0.03 & 0.03 & 0.60 & 0.39 & 0.31 & 0.06 & 0.05 & 0.04 & 0.08 & 0.05 & 0.06 \\
\hline C.D. @ 1 \% & 0.12 & 0.12 & 0.12 & NS & NS & NS & 0.17 & 0.15 & 0.12 & 0.31 & 0.19 & 0.21 \\
\hline \multicolumn{13}{|l|}{ Foliar spray (T) } \\
\hline $\mathbf{T}_{1}$ & 13.35 & 13.21 & 13.28 & 94.26 & 92.93 & 93.59 & 16.85 & 16.26 & 16.56 & 15.78 & 15.53 & 15.66 \\
\hline $\mathbf{T}_{2}$ & 13.44 & 13.30 & 13.37 & 95.23 & 94.06 & 94.64 & 17.05 & 16.98 & 17.02 & 16.74 & 16.55 & 16.65 \\
\hline $\mathbf{T}_{3}$ & 13.51 & 13.34 & 13.43 & 95.53 & 94.26 & 94.90 & 17.06 & 16.98 & 17.02 & 16.78 & 16.65 & 16.71 \\
\hline $\mathbf{T}_{4}$ & 13.54 & 13.38 & 13.46 & 95.99 & 94.60 & 95.29 & 17.08 & 17.02 & 17.05 & 16.80 & 16.73 & 16.77 \\
\hline $\mathbf{T}_{5}$ & 13.40 & 13.24 & 13.32 & 94.69 & 93.43 & 94.06 & 16.98 & 16.66 & 16.82 & 16.66 & 16.60 & 16.63 \\
\hline $\mathbf{T}_{6}$ & 13.59 & 13.45 & 13.52 & 96.22 & 94.90 & 95.56 & 17.34 & 17.44 & 17.39 & 17.39 & 17.37 & 17.38 \\
\hline $\mathbf{T}_{7}$ & 13.59 & 13.45 & 13.52 & 96.18 & 94.86 & 95.52 & 17.43 & 17.51 & 17.47 & 17.32 & 17.30 & 17.31 \\
\hline $\mathbf{T}_{8}$ & 13.61 & 13.47 & 13.54 & 96.90 & 95.66 & 96.28 & 17.28 & 17.41 & 17.35 & 17.54 & 17.42 & 17.48 \\
\hline S. Em. \pm & 0.05 & 0.05 & 0.05 & 0.98 & 0.64 & 0.51 & 0.10 & 0.09 & 0.07 & 0.13 & 0.08 & 0.09 \\
\hline C.D. @ $1 \%$ & 0.20 & 0.19 & 0.20 & NS & NS & 1.945 & 0.28 & 0.25 & 0.19 & 0.51 & 0.31 & 0.34 \\
\hline \multicolumn{13}{|l|}{ Interactions (D X T) } \\
\hline $\mathrm{D}_{1} \mathrm{~T}_{\mathrm{I}}$ & 13.61 & 13.51 & 13.56 & 94.90 & 93.09 & 93.99 & 16.99 & 16.91 & 16.95 & 16.25 & 16.02 & 16.14 \\
\hline $\mathrm{D}_{1} \mathrm{~T}_{2}$ & 13.69 & 13.58 & 13.64 & 95.70 & 94.00 & 94.85 & 17.20 & 17.30 & 17.25 & 16.91 & 16.80 & 16.86 \\
\hline $\mathrm{D}_{1} \mathrm{~T}_{3}$ & 13.74 & 13.61 & 13.68 & 96.00 & 94.40 & 95.20 & 17.37 & 17.33 & 17.35 & 16.93 & 16.87 & 16.90 \\
\hline $\mathrm{D}_{1} \mathrm{~T}_{4}$ & 13.77 & 13.65 & 13.71 & 96.30 & 94.80 & 95.55 & 17.25 & 17.40 & 17.33 & 16.95 & 16.90 & 16.93 \\
\hline $\mathrm{D}_{1} \mathrm{~T}_{5}$ & 13.65 & 13.54 & 13.60 & 95.30 & 93.60 & 94.45 & 16.98 & 16.81 & 16.90 & 16.85 & 16.71 & 16.78 \\
\hline $\mathrm{D}_{1} \mathrm{~T}_{6}$ & 13.81 & 13.74 & 13.78 & 96.50 & 95.10 & 95.80 & 17.29 & 17.62 & 17.46 & 17.96 & 17.87 & 17.92 \\
\hline $\mathbf{D}_{1} \mathbf{T}_{7}$ & 13.81 & 13.75 & 13.78 & 96.40 & 95.10 & 95.75 & 18.04 & 17.83 & 17.94 & 17.29 & 17.67 & 17.48 \\
\hline $\mathrm{D}_{1} \mathrm{~T}_{8}$ & 13.83 & 13.77 & 13.80 & 97.00 & 95.80 & 96.40 & 18.06 & 17.83 & 17.95 & 17.32 & 17.67 & 17.50 \\
\hline $\mathbf{D}_{2} \mathbf{T}_{1}$ & 13.40 & 13.26 & 13.33 & 94.19 & 93.00 & 93.59 & 16.80 & 16.01 & 16.41 & 16.15 & 16.00 & 16.08 \\
\hline $\mathrm{D}_{2} \mathrm{~T}_{2}$ & 13.49 & 13.36 & 13.42 & 95.19 & 93.70 & 94.44 & 17.00 & 16.92 & 16.96 & 16.80 & 16.65 & 16.73 \\
\hline $\mathrm{D}_{2} \mathrm{~T}_{3}$ & 13.54 & 13.38 & 13.46 & 95.60 & 94.30 & 94.95 & 16.95 & 16.92 & 16.94 & 16.85 & 16.74 & 16.80 \\
\hline $\mathrm{D}_{2} \mathrm{~T}_{4}$ & 13.54 & 13.44 & 13.49 & 95.97 & 94.60 & 95.28 & 17.15 & 17.01 & 17.08 & 16.90 & 16.79 & 16.84 \\
\hline $\mathbf{D}_{2} \mathbf{T}_{5}$ & 13.46 & 13.28 & 13.37 & 94.69 & 93.40 & 94.04 & 16.99 & 16.87 & 16.93 & 16.77 & 16.60 & 16.69 \\
\hline $\mathrm{D}_{2} \mathrm{~T}_{6}$ & 13.60 & 13.50 & 13.55 & 96.18 & 94.90 & 95.54 & 17.93 & 17.71 & 17.82 & 17.30 & 17.52 & 17.41 \\
\hline $\mathbf{D}_{2} \mathbf{T}_{7}$ & 13.60 & 13.50 & 13.55 & 96.15 & 94.80 & 95.47 & 17.31 & 17.71 & 17.51 & 17.91 & 17.51 & 17.71 \\
\hline $\mathrm{D}_{2} \mathrm{~T}_{8}$ & 13.62 & 13.51 & 13.56 & 97.00 & 95.70 & 96.35 & 17.33 & 17.61 & 17.47 & 17.94 & 17.63 & 17.79 \\
\hline $\mathrm{D}_{3} \mathrm{~T}_{1}$ & 13.04 & 12.88 & 12.96 & 93.70 & 92.70 & 93.20 & 16.76 & 15.86 & 16.31 & 14.95 & 14.56 & 14.76 \\
\hline $\mathrm{D}_{3} \mathrm{~T}_{2}$ & 13.16 & 12.97 & 13.06 & 94.80 & 94.50 & 94.65 & 16.95 & 16.72 & 16.84 & 16.51 & 16.21 & 16.36 \\
\hline $\mathrm{D}_{3} \mathrm{~T}_{3}$ & 13.27 & 13.02 & 13.14 & 95.00 & 94.10 & 94.55 & 16.85 & 16.70 & 16.77 & 16.55 & 16.33 & 16.44 \\
\hline $\mathrm{D}_{3} \mathrm{~T}_{4}$ & 13.31 & 13.06 & 13.18 & 95.69 & 94.40 & 95.04 & 16.85 & 16.65 & 16.75 & 16.56 & 16.49 & 16.53 \\
\hline $\mathrm{D}_{3} \mathrm{~T}_{5}$ & 13.10 & 12.92 & 13.01 & 94.10 & 93.29 & 93.69 & 16.95 & 16.30 & 16.63 & 16.35 & 16.48 & 16.42 \\
\hline $\mathrm{D}_{3} \mathrm{~T}_{6}$ & 13.37 & 13.11 & 13.24 & 95.99 & 94.70 & 95.35 & 16.80 & 16.99 & 16.90 & 16.90 & 16.71 & 16.81 \\
\hline $\mathbf{D}_{3} \mathbf{T}_{7}$ & 13.36 & 13.11 & 13.24 & 96.00 & 94.70 & 95.35 & 16.96 & 16.98 & 16.97 & 16.75 & 16.72 & 16.73 \\
\hline $\mathrm{D}_{3} \mathrm{~T}_{8}$ & 13.38 & 13.14 & 13.26 & 96.69 & 95.50 & 96.09 & 16.44 & 16.80 & 16.62 & 17.35 & 16.94 & 17.15 \\
\hline S. Em. \pm & 0.09 & 0.09 & 0.09 & 1.70 & 1.11 & 0.88 & 0.17 & 0.15 & 0.12 & 0.23 & 0.14 & 0.16 \\
\hline C.D. @1\% & 0.35 & 0.34 & 0.34 & NS & NS & 3.36 & 0.49 & 0.43 & 0.34 & 0.88 & 0.54 & 0.60 \\
\hline
\end{tabular}


Table.3 Effect of date of sowing and foliar application of nutrients on seedling dry weight, seedling vigour index-I, germination rate index and seed infection of soybean

\begin{tabular}{|c|c|c|c|c|c|c|c|c|c|c|c|c|}
\hline \multirow{2}{*}{$\begin{array}{l}\text { Treatments } \\
\text { Dates of sowing (D) }\end{array}$} & \multicolumn{3}{|c|}{ Seedling dry weight (mg) } & \multicolumn{3}{|c|}{ Seedling vigor index -I } & \multicolumn{3}{|c|}{ Germination rate index } & \multicolumn{3}{|c|}{ Seed infection (\%) } \\
\hline & 2016 & 2017 & Pooled & 2016 & 2017 & pooled & 2016 & 2017 & pooled & 2016 & 2017 & pooled \\
\hline $\mathbf{D}_{1}$ & 124.0 & 121.9 & 124.0 & 3309 & 3255 & 3282 & 64.09 & 64.01 & 64.05 & 13.54 & 14.94 & 14.24 \\
\hline $\mathbf{D}_{2}$ & 123.4 & 120.4 & 123.0 & 3277 & 3209 & 3243 & 63.93 & 63.88 & 63.91 & 19.24 & 20.15 & 19.69 \\
\hline $\mathbf{D}_{3}$ & 121.3 & 117.2 & 120.3 & 3173 & 3104 & 3139 & 63.82 & 63.64 & 63.73 & 21.38 & 23.51 & 22.45 \\
\hline S. Em. \pm & 0.7 & 0.6 & 0.6 & 17 & 11 & 9 & 0.03 & 0.04 & 0.03 & 0.16 & 0.17 & 0.17 \\
\hline C.D. @ $1 \%$ & 1.9 & 1.7 & 1.7 & 49 & 31 & 25 & 0.07 & 0.13 & 0.07 & 0.46 & 0.50 & 0.48 \\
\hline \multicolumn{13}{|l|}{ Foliar spray (T) } \\
\hline $\mathrm{T}_{1}$ & 121.3 & 118.4 & 120.9 & 3077 & 2954 & 3016 & 63.78 & 63.70 & 63.74 & 20.68 & 22.75 & 21.71 \\
\hline $\mathbf{T}_{2}$ & 122.3 & 119.1 & 121.8 & 3218 & 3154 & 3186 & 63.87 & 63.79 & 63.83 & 18.88 & 20.67 & 19.77 \\
\hline $\mathbf{T}_{3}$ & 122.6 & 119.7 & 122.2 & 3232 & 3170 & 3201 & 63.90 & 63.82 & 63.86 & 18.27 & 19.52 & 18.89 \\
\hline$T_{4}$ & 123.1 & 120.2 & 122.7 & 3253 & 3193 & 3223 & 63.93 & 63.85 & 63.89 & 17.63 & 18.48 & 18.05 \\
\hline$T_{5}$ & 121.9 & 118.8 & 121.4 & 3185 & 3107 & 3146 & 63.81 & 63.74 & 63.78 & 19.91 & 21.57 & 20.74 \\
\hline$T_{6}$ & 123.8 & 120.7 & 123.3 & 3342 & 3303 & 3323 & 64.06 & 63.92 & 63.99 & 16.36 & 17.77 & 17.07 \\
\hline $\mathbf{T}_{7}$ & 123.8 & 120.7 & 123.3 & 3343 & 3302 & 3322 & 64.06 & 63.95 & 64.01 & 16.37 & 17.77 & 17.07 \\
\hline$T_{8}$ & 124.2 & 121.1 & 123.7 & 3374 & 3332 & 3353 & 64.17 & 64.00 & 64.08 & 16.32 & 17.73 & 17.03 \\
\hline S. Em. \pm & 1.1 & 1.0 & 1.0 & 28 & 18 & 14 & 0.04 & 0.07 & 0.04 & 0.26 & 0.29 & 0.27 \\
\hline C.D. @ $1 \%$ & NS & NS & NS & 79 & 50 & 41 & 0.12 & NS & 0.12 & 0.75 & 0.81 & 0.78 \\
\hline \multicolumn{13}{|l|}{ Interactions (D X T) } \\
\hline $\mathbf{D}_{1} \mathbf{T}_{\mathrm{I}}$ & 122.1 & 119.6 & 121.9 & 3155 & 3066 & 3110 & 63.85 & 63.81 & 63.83 & 15.96 & 17.88 & 16.92 \\
\hline $\mathbf{D}_{1} \mathbf{T}_{2}$ & 123.1 & 121.2 & 123.2 & 3264 & 3205 & 3234 & 63.93 & 63.91 & 63.92 & 14.32 & 15.64 & 14.98 \\
\hline$D_{1} T_{3}$ & 123.6 & 121.7 & 123.7 & 3292 & 3228 & 3260 & 63.96 & 63.95 & 63.95 & 13.40 & 14.88 & 14.14 \\
\hline $\mathbf{D}_{1} \mathbf{T}_{4}$ & 124.1 & 122.3 & 124.2 & 3293 & 3252 & 3273 & 63.98 & 63.96 & 63.97 & 13.20 & 14.04 & 13.62 \\
\hline$D_{1} T_{5}$ & 122.6 & 120.9 & 122.8 & 3223 & 3137 & 3180 & 63.89 & 63.86 & 63.88 & 15.24 & 16.08 & 15.66 \\
\hline$D_{1} T_{6}$ & 125.3 & 122.9 & 125.2 & 3403 & 3375 & 3389 & 64.30 & 64.10 & 64.20 & 12.08 & 13.68 & 12.88 \\
\hline $\mathbf{D}_{1} \mathbf{T}_{7}$ & 125.4 & 123.0 & 125.3 & 3406 & 3376 & 3391 & 64.30 & 64.20 & 64.25 & 12.08 & 13.68 & 12.88 \\
\hline $\mathrm{D}_{1} \mathrm{~T}_{8}$ & 125.8 & 123.2 & 125.6 & 3432 & 3401 & 3417 & 64.50 & 64.31 & 64.40 & 12.00 & 13.64 & 12.82 \\
\hline$D_{2} T_{1}$ & 121.9 & 119.5 & 121.8 & 3105 & 2976 & 3041 & 63.80 & 63.77 & 63.79 & 22.04 & 23.52 & 22.78 \\
\hline $\mathrm{D}_{2} \mathrm{~T}_{2}$ & 123.1 & 119.5 & 122.3 & 3218 & 3145 & 3181 & 63.88 & 63.86 & 63.87 & 20.20 & 21.52 & 20.86 \\
\hline$D_{2} T_{3}$ & 123.2 & 120.1 & 122.7 & 3231 & 3174 & 3203 & 63.92 & 63.88 & 63.90 & 19.68 & 20.04 & 19.86 \\
\hline$D_{2} T_{4}$ & 123.7 & 120.6 & 123.2 & 3269 & 3197 & 3233 & 63.95 & 63.90 & 63.92 & 18.84 & 19.16 & 19.00 \\
\hline$D_{2} T_{5}$ & 122.6 & 119.2 & 122.0 & 3197 & 3126 & 3161 & 63.83 & 63.81 & 63.82 & 21.20 & 22.76 & 21.98 \\
\hline$D_{2} T_{6}$ & 124.1 & 121.4 & 123.8 & 3388 & 3344 & 3366 & 63.99 & 63.95 & 63.97 & 17.32 & 18.08 & 17.70 \\
\hline $\mathbf{D}_{2} \mathbf{T}_{7}$ & 124.0 & 121.3 & 123.7 & 3386 & 3339 & 3362 & 63.98 & 63.95 & 63.97 & 17.32 & 18.08 & 17.70 \\
\hline $\mathbf{D}_{2} \mathbf{T}_{\mathbf{8}}$ & 124.5 & 121.8 & 124.2 & 3422 & 3373 & 3398 & 64.10 & 63.96 & 64.03 & 17.28 & 18.04 & 17.66 \\
\hline$D_{3} T_{1}$ & 120.0 & 116.0 & 119.0 & 2971 & 2821 & 2896 & 63.68 & 63.50 & 63.59 & 24.04 & 26.84 & 25.44 \\
\hline$D_{3} T_{2}$ & 120.8 & 116.6 & 119.8 & 3173 & 3112 & 3142 & 63.79 & 63.61 & 63.70 & 22.12 & 24.84 & 23.48 \\
\hline$D_{3} T_{3}$ & 121.1 & 117.2 & 120.2 & 3173 & 3108 & 3140 & 63.82 & 63.64 & 63.73 & 21.72 & 23.64 & 22.68 \\
\hline$D_{3} T_{4}$ & 121.4 & 117.7 & 120.6 & 3197 & 3128 & 3163 & 63.85 & 63.68 & 63.77 & 20.84 & 22.24 & 21.54 \\
\hline$D_{3} T_{5}$ & 120.3 & 116.3 & 119.4 & 3135 & 3059 & 3097 & 63.72 & 63.56 & 63.64 & 23.28 & 25.88 & 24.58 \\
\hline$D_{3} T_{6}$ & 122.1 & 117.9 & 121.1 & 3236 & 3192 & 3214 & 63.89 & 63.71 & 63.80 & 19.68 & 21.56 & 20.62 \\
\hline $\mathbf{D}_{3} \mathbf{T}_{7}$ & 122.1 & 117.9 & 121.1 & 3236 & 3191 & 3214 & 63.90 & 63.70 & 63.80 & 19.72 & 21.56 & 20.64 \\
\hline$D_{3} T_{8}$ & 122.4 & 118.3 & 121.4 & 3267 & 3222 & 3244 & 63.91 & 63.72 & 63.82 & 19.68 & 21.52 & 20.60 \\
\hline S. Em. \pm & 1.9 & 1.7 & 1.7 & 48 & 31 & 25 & 0.07 & 0.13 & 0.07 & 0.46 & 0.49 & 0.48 \\
\hline C.D. @ 1 \% & NS & 4.9 & 4.7 & 137 & 87 & 71 & 0.21 & 0.36 & 0.21 & 1.30 & 1.41 & 1.35 \\
\hline
\end{tabular}


Higher seedling dry weight (124 mg) in early sowings might be due to larger seed size which could be attributed to more food reserves in the seed ultimately resulting into good seedlings. Decrease in seedling dry weight was due to nutrients supply from mother plant was restricted to seed due to disruption of vascular connection and utilization in various physiological and metabolic processes (Khatun et al., 2009). These findings are supported by Khan (2001) who reported that soybean seedling dry weight decreased in seeds from late sown crop. Kasul et al., (2003) reported a significant positive correlation between weight of seed and seedling vigour and seedling dry weight.

The vigour index-I of soybean differs significantly due to different date of sowing. There was significant reduction in vigour index from June first fortnight $\left(\mathrm{D}_{1}\right)$ to July first fortnight $\left(\mathrm{D}_{3}\right)$ with maximum (3308, 3255 and 3282) observed at $D_{1}$ date of sowing and minimum (3173, 3104 and 3139) recorded at $\mathrm{D}_{3}$ date of sowing during 2016, 2017 and pooled data, respectively (Table 3). The variation in seed germination and vigour comes from the environmental conditions that the crop experiences during the seed development and maturation (Dornbos, 1995). These findings are in line with the reports of Rahman et al., (2013) who reported that seeds from optimum time of sowing had high seedling vigour index because of high seed quality.

Seeds from June first fortnight $\left(\mathrm{D}_{1}\right)$ sowing recorded significantly highest Germination rate index $(64.09,64.01$ and 64.05) followed by June second fortnight $\left(\mathrm{D}_{2}\right)$ of sowing (63.93, 63.88 and 63.91) as compared to lower Germination rate index $(63.82,63.64$ and 63.73) was recorded in July first fortnight $\left(D_{3}\right)$ of sowing during 2016, 2017 and pooled data, respectively (Table 3). Speed of germination was found to be better from seeds obtained from June first fortnight sowings. This might be due to favourable environmental conditions during seed development, high initial seed quality and higher seed size resulting into higher seed germination and seed vigour. These findings are supported by Rahman et al., (2013) who concluded that seed produced from early sowings had higher seed quality and vigour index than seed produced from delayed sowings.

Results on seed infection (\%) showed that there was a significant difference among seed lots harvested from crop sown on different sowing dates (Table 3). Maximum seed infection was recorded in July first fortnight $\left(D_{3}\right)$ of sowing $(21.38,23.51$ and $22.45 \%)$ and lowest infection (13.54, 14.94 and 14.24 $\%$ ) was recorded in seeds harvested from first fortnight of sowing $\left(D_{1}\right)$ during 2016, 2017 and pooled data, respectively. Seed infection was more observed in third date of sowing (22.45 \%). This might be due to seeds obtained from these sowings had relatively higher moisture content and fungal infection. These results are in contrast with findings of Khalil et al., (2001) who noticed that seeds received from early sowings had lower germination and higher infection of seed borne pathogens while, seeds from delayed sowings produced smaller seeds with higher germination and little infection with seed borne pathogens. The contrasting results might be due to differences in the weather parameters prevailed at seed maturity and harvesting.

There was significant reduction in dehydrogenase activity from June first fortnight $\left(\mathrm{D}_{1}\right)$ to July first fortnight $\left(\mathrm{D}_{3}\right)$ with maximum dehydrogenase activity (0.616, 0.612 and 0.614 ) observed at $\mathrm{D}_{1}$ date of sowing and minimum (0.584, 0.569 and 0.577 ) recorded at $\mathrm{D}_{3}$ date of sowing during 
2016, 2017 and pooled data, respectively (Fig. 1). This might be due to high initial seed quality. These results are in conformity with Madhavi, et al., (2000). They stated that, the differences will show clearly at the maturity phase which early planting date affected the rapidly increasing the activity of dehydrogenase enzyme whereas late planting shoe slower trend.

Seeds from June first fortnight $\left(D_{1}\right)$ sowing recorded significantly higher $(18.15,18.11$ and $18.13 \%)$ oil content followed by June second fortnight $\left(\mathrm{D}_{2}\right)$ of sowing $(18.10,17.95$ and $18.03 \%)$ as compared to lower (17.94, 17.85 and $17.90 \%$ ) oil content was recorded in July first fortnight $\left(\mathrm{D}_{3}\right)$ of sowing. Higher Protein content was recorded in seeds sown on first fortnight of July $\left(\mathrm{D}_{3}\right)(38.04,37.95$ and $37.99 \%$ ) followed by second fortnight of June $\left(\mathrm{D}_{2}\right)$ recorded $37.93,37.81$ and 37.87 per cent while, lower Protein content was recorded in plots sown on first fortnight of June $\left(\mathrm{D}_{1}\right)(37.65,37.52$ and $37.59 \%)$ during 2016, 2017 and pooled data, respectively. These results are in line with the reports of Bachlava and Cardinal (2009), this was mainly due to the effect of temperature on the enzymes controlling biosynthesis of soybean seed fatty acids, especially at a seed- fill stage.

Foliar application of nutrients exhibited consistent and significant difference on test weight, germination per cent, root length, shoot length, seedling dry weight, seedling vigour index- I, germination rate index, total dehydrogenase activity, oil and protein, highest values was recorded in $\mathrm{KNO}_{3} @ 0.5$ $\%+\mathrm{KH}_{2} \mathrm{PO} 4 @ 0.5 \%+$ Boron $0.50 \%$ (13.54 g, $96.28 \%, 17.35 \mathrm{~cm}, 17.48 \mathrm{~cm}, 123.7$ $\mathrm{mg}, 3353,64.08,0.606$ OD value, $18.10 \%$ and $37.93 \%$ ) which was on par with (T6) 19:19:19@3\%+Boron@ 0.50\% (13.52g, $95.56 \%, 17.39 \mathrm{~cm}, 17.38 \mathrm{~cm}, 123.3 \mathrm{mg}, 3323$, $63.99,0.605$ OD value, $18.07 \%$ and $37.91 \%$ ) and $\left(\mathrm{T}_{7}\right) \mathrm{KNO}_{3} @ 1 \%+\mathrm{KH}_{2} \mathrm{PO}_{4} @ 0.5 \%$ $(13.52 \mathrm{~g}, 95.52 \%, 17.47 \mathrm{~cm}, 17.31 \mathrm{~cm}, 123.3$ $\mathrm{mg}, 3322,64.01,0.605$ OD value, $18.07 \%$ and $37.91 \%)$. The least $(13.28 \mathrm{~g}, 93.59 \%$, $16.56 \mathrm{~cm}, 15.66 \mathrm{~cm}, 120.9 \mathrm{mg}, 3016,63.74$, 0.576 OD value, $17.91 \%$ and $37.66 \%$ ) values was recorded in control $\left(\mathrm{T}_{1}\right)$ based on pooled data (Table 2 and 3 and Fig. 1 and 2). This might be due foliar spray of water soluble fertilizers containing major nutrients at critical growth stages might have resulted in quick and spot availability of optimum quantity of nutrients in required form to the soybean crop. This resulted in better absorption of nutrients by leaves in soybean which might have helped in the production of more number of flowers and pods per plant, better seed setting, more number of developed seeds ultimately higher seed weight. So significantly highest seed weight were recorded in treated plots receiving 19:19:19 and $\mathrm{KNO}_{3} @ 0.5 \%+\mathrm{KH}_{2} \mathrm{PO}_{4} @ 0.5 \%+$ Boron $0.5 \%$. The presence of potassium in 19:19:19 helps in higher supply and absorption of nitrogen by soybean leaves leading to increased chlorophyll synthesis and photosynthesis might have translocated to the developing seeds. The investigations were in accordance with the finding of Vinod Kumar et al., (2013) in soybean. Foliar application of water soluble fertilizer at pod development stage. Since, the higher nitrogen supply through foliar application at different crop growth stages resulted in enhancement of protein content of seeds, suggesting that hydrocarbons synthesized during photosynthetic process are diverted to form more of proteins (Chaurasia et al., 2005, Venkatesh and Basu, 2011). The increase in protein was also accompanied by increase in $\mathrm{K}$ and $\mathrm{N}$ in leaf tissues. Our findings were in agreement with earlier studies (Bellaloui, et al., 2010, Soybean) where they reported an increase in protein with higher $\mathrm{K}$ levels. Potassium is directly or indirectly involved in plant protein metabolism with $\mathrm{K}$ crucial in 
most steps of protein synthesis (Blevins, 2010, other crops). Previous research reported enhanced transport of essential amino acids at higher $\mathrm{K}$ fertilizer levels, especially to the developing seeds (Mengel et al., 1991 other crops).

Thus from the experiment it could be concluded that with delayed sowing, seed quality attributes were adversely affected. Small fluctuations in the weather (temperature) showed higher variations in plant growth and development, which finally influenced on the yield and quality of soybean. Considering the changes in plant growth, seed yield and quality, first fortnight of June sowing sprayed with $\mathrm{KNO}_{3} @ 0.5 \%$ + $\mathrm{KH}_{2} \mathrm{PO}_{4} @ 0.5 \%+$ Boron $0.50 \%$ and also 19: 19: $19 @ 3 \%+$ Boron @ $0.50 \%$ maintained better crop growth, seed yield and quality of soybean.

\section{References}

Alexandrov, V. A. and Hoogenboom, G., 2001, Climate variation and crop production in Georgia, USA during the twentieth century. Climate Res., 17(1): 33-43.

Anonymous, 2017, USDA, Foreign Agricultural Services, Washimgton, DC.

Bachlava, E, and Cardinal, A.J., 2009, Correlation between temperature and oleic acid seed content in three segregating soybean populations. Crop Sci., 49(4):1328-1335.

Bastidas, A. M., Setryono, T. D., Dobermann, A., Cassman, K.G., Elmore, R. W., Graef, G. L. and Specht, J. E., 2008, Soybean sowing date: The vegetative, reproductive and agronomic impacts. Crop Sci., 48: 727-740.

Bellaloui, N., Bruns, H.A., Gillen, A.M., Abbas, H.K., Zablotowicz, R.M., Mengistu, A. and Paris, R.L., 2010,
Soybean Seed Protein, Oil, Fatty Acids, and Mineral Composition as Influenced by Soybean-Corn Rotation. Agricul Sci., 1:102-109.

Blevins, D.G., 2010, Role of Potassium in Protein Metabolism in Plants. In: Munson, R.D., Ed., Potassium in Agriculture, ASA, CSSA and SSSA, Madison, 413-424.

Chaurasia, S. N. S., Singh, K. P. and Mathura, Rai, 2005, Effect of foliar application of water soluble fertilizers on growth, yield and quality of tomato (Lycopersicon esculentum L.). Sri Lankan J. Agric. Sci., 42: 66 - 70.

Dornbos, D.L., 1995, Production environment and seed quality. In: Seed Quality. Basic Mechanisms and Agricultural Implication, Food Product Press, New York. 119-152.

Jayabal, A., Revathy, M. and Saxena, M. G., 1999, Effect of foliar nutrition on nutrient uptake pattern in soybean. Andhra Agric. J., 46: 243-244.

Kasul, R.T., Kadam, N.R., Pavithrakar, N.R and Khedekar, R.P., 2003, Improvement of seed quality in the marginal seed lots of cotton by using specific gravity separator. Seed Res., 31(2): 213-218.

Khalil, S.K., Maxal, J.G and Murray, L.W., 2001, Soybean seed matured on different dates affect seed quality. Pakisthan J. of Biol Sci., 4: 365-370.

Khan, A.Z., 2001, Influence of planting date and plant density on canopy temperature, seed development, seed quality and yield of soybean. Ph.D Dissertation, University of Agriculture, Faisalabad.

Khan, A.Z., 2001, Influence of planting date and plant density on canopy temperature, seed development, seed quality and yield of soybean. Ph.D Dissertation, University of Agriculture, Faisalabad. 
Khatun, A., Kabir, G and. Bhuiyan, M.A.H., 2009, Effect of harvesting stages on the seed quality of lentil (Lens culinaris L.) during storage. Bangladesh J of Agricul Res., 34 (4): 565-576.

Kumar, B.N.M., Basavegowda, Vyakaranahal, B.S., Deshpande, V.K and Kenchanagoudar, P.V., 2011, Influence of sowing dates on production of seed yield in niger (Guizotia abyssinica Cass.). Karnataka J. of Agricul Sci. 24(3): 289-293.

Madhavi, P., Ramaiah, N. V., Satyanarayana, V. and Vijaykumar, B., 2000, Effect of sowing dates and fertilizer levels on yield and quality of soybean under Telangana region of Andhra Pradesh. The J. of Res ANGRAU, 29(4): 92-94.

Mengel, K., Secer, M. and Koch, K., 1991, Potassium Effect on Protein Formation and Amino Acid Grain. Agronomy J., 73, 74-78.

Rahman, M. M., Hampton, J.G. and Hill, M.J., 2006, Soybean seed yield as affected by time of sowing in a cool temperature environment. $J$. of $\mathrm{New}$ Seeds., 7: 1-15.

Rahman, M.M., Rahman, M.M. and Hussain, M.M., 2013, Effect of sowing date on germination and vigour of soybean
(Glycine max) seeds. The Agriculturists. 11 (1): 67-75.

Uem and Unioeste, 2003, Sowing seasons and quality of soybean seeds. Scientia Agricola. 60: 245-252.

Venkatesh, M. S. and Basu, P. S., 2011, Effect of foliar application of urea on growth, yield and quality of chickpea under rainfed conditions. J. Food legume, 24(2): 110-112.

Vibhute, C. P., 1998, A process for manufacturing complex solid and liquid completely water soluble fertilizer. Ferti. News, 43(8): 63-69.

Vinod kumar C, Vaijapuri K, Mohamed Amanullah M, Gopalaswamy G, 2013, Influence of foliar spray of nutrients on yield and economics of soybean. J. Biol. Sci., 13:563-565.

Yari, V., Frnia, A., Maleki, A., Moradi, M., Naseri, R., Ghasemi, M. and Lotfi, A., 2013, Yield and yield components of soybean cultivars as affected by planting date. Bulletin of Environment, Pharmacology and Life Sci., 2(7): 8590.

Zayed, B.A., Salem, A. and El, Sharkawy, H.M., 2011, Effect of different micronutrient treatments on rice (Oryza sativa L.) growth and yield under saline soil conditions. World J. of Agricul Sci., 7 (2): 179-184.

\section{How to cite this article:}

Sumalatha, G.M. and Uppar, D.S. 2018. Effect of Date of Sowing and Foliar Application of Nutrients on Seed Quality of Soybean. Int.J.Curr.Microbiol.App.Sci. 7(08): 535-544. doi: https://doi.org/10.20546/ijcmas.2018.708.059 